\title{
Aromatase knockout mice reveal an impact of estrogen on drug-induced alternation of murine electrocardiography parameters
}

\author{
Junko Kurokawa ${ }^{1, *}$, Tetsuo Sasano ${ }^{1, *}$, Masami Kodama ${ }^{1}$, Min Li$^{1}$, Yusuke Ebana ${ }^{1}$, \\ Nobuhiro Harada ${ }^{3}$, Shin-ichiro Honda ${ }^{3}$, Haruaki Nakaya ${ }^{2}$ and Tetsushi Furukawa ${ }^{1}$ \\ ${ }^{1}$ Department of Bio-Informational Pharmacology, Medical Research Institute, Tokyo Medical Dental University, \\ 1-5-45 Yushima, Bunkyo-ku, Tokyo 113-8510, Japan \\ ${ }^{2}$ Department of Pharmacology, Chiba University Graduate School of Medicine, \\ 1-8-1 Inohana, Chuo-ku, Chiba 260-8670, Japan \\ ${ }^{3}$ Department of Biochemistry, School of Medicine, Fujita Health University, \\ 1-98 Dengakugakubo, Kutsukake-cho, Toyoake, Aichi 470-1192, Japan
}

(Received January 28, 2015; Accepted March 4, 2015)

\begin{abstract}
Our in vitro characterization showed that physiological concentrations of estrogen partially suppressed the $\mathrm{I}_{\mathrm{Kr}}$ channel current in guinea pig ventricular myocytes and the human ether-a-gogo-related gene (hERG) channel currents in $\mathrm{CHO}-\mathrm{K} 1$ cells regardless of estrogen receptor signaling and revealed that the partially suppressed hERG currents enhanced the sensitivity to the hERG blocker E-4031. To obtain in vivo proof-of-concept data to support the effects of estrogen on cardiac electrophysiology, we here employed an aromatase knockout mouse as an in vivo estrogen-null model and compared the acute effects of E-4031 on cardiac electrophysiological parameters with those in wild-type mice (C57/ BL6J) by recording surface electrocardiogram (ECG). The ablation of circulating estrogens blunted the effects of E-4031 on heart rate and QT interval in mice under a denervation condition. Our result provides in vivo proof of principle and demonstrates that endogenous estrogens increase the sensitivity of E-4031 to cardiac electrophysiology.
\end{abstract}

Key words: Estrogen, hERG channel, Cardiac electrophysiology, Cardiac toxicology, ECG

\section{INTRODUCTION}

It is widely accepted that women are more prone to developing drug-induced arrhythmias (torsade de pointes, $\mathrm{TdP}$ ) in association with prolongation of QT intervals caused by blockade of human ether-a-go-go-related gene (hERG) currents (Makkar et al., 1993). Clinical evidence implies that sex hormones appear to play important roles in sex differences in baseline QT intervals and pro-arrhythmias (Kurokawa et al., 2012). We found that physiological serum levels of $17 \beta$-estradiol (E2) and estrone 3-sulfate acutely prolonged action potential durations and suppressed guinea pig $\mathrm{I}_{\mathrm{Kr}}$ channel currents $\left(I_{\mathrm{Kr}}\right)$ and hERG currents (Kurokawa et al., 2008; Kakusaka et al., 2009). The effects of estrogens on $I_{\mathrm{Kr}}$ or hERG currents are independent of estrogen receptor signaling, where- as the direct interaction between the aromatic centroid of estrogen and the side chain of F656 hERG suppresses hERG currents. Furthermore, E2 augmented the hERG blockade by E-4031, the binding site of which includes F656, and increased the sensitivity of E-4031 to $\mathrm{QT}_{\mathrm{C}}$ prolongation in the Langendorff-perfused heart (Kurokawa et al., 2008). Thus, these in vitro data strongly suggest that the direct binding of estrogen to the $\mathrm{hERG}$ channel results in hERG suppression and increased E-4031 sensitivity. These data are consistent with the previously shown E2-induced delay of cardiac repolarization in rabbits and ibutilide-induced $\mathrm{QT}_{\mathrm{C}}$ prolongation in the late follicular phase of women (Kurokawa and Furukawa, 2013; Hara et al., 1998; Rodriguez et al., 2001). However, there is no in vivo proof-of-concept that supports the impact of estrogen-induced hERG suppression on repolarization process

Correspondence: Junko Kurokawa (E-mail: junkokuro.bip@mri.tmd.ac.jp)

*These authors equally contributed to this work. 


\section{J. Kurokawa et al.}

of the heart. To show the influence of estrogens on hERG blocker sensitivity in vivo, here we employed an aromatase cytochrome P450 knockout (ArKO) mouse that is deficient in aromatase activity by a targeted disruption of the CYP19 gene (Honda et al., 1998). Aromatase metabolizes testosterone to E2 and contributes to local estrogen synthesis including in the murine heart (Jazbutyte et al., 2012). ArKO mice cannot aromatize androgen to estrogen; therefore, we tested the influence of local estrogens on electrocardiography (ECG) parameters and the sensitivity to the selective hERG blocker E-4031 in vivo.

\section{MATERIALS AND METHODS}

The methods used here are described in detail elsewhere (Suzuki et al., 2002; Honda et al., 1998; Miki et al., 2002; Kuma et al., 2004).

\section{Animals}

This study was conducted in strict accordance with the recommendations of the Guide for the Care and Use of Laboratory Animals of the National Institutes of Health. The protocol was approved by the Committees on the Ethics of Animal Experiments of Tokyo Medical and Dental University (0140103A), Chiba University (20060021), and Fujita Health University (M1901). An ArKO mouse line was generated as described previously (Honda et al., 1998). We generated 12 homozygous ArKO female mice and then used them at 2-3 months of age. Two heterozygous ArKO mice were used to check ion channel expression in the ArKO mouse line in Fig. 1. The data from three of the homozygous ArKO mice were omitted because of unstable baseline ECG recordings (checked individually by four investigators). Thus, data from nine of 12 homozygous ArKO mice were intensively analyzed. We also used seven wild-type female mice (2-3 months of age) at the late-proestrus stages, which included five female littermates. Because estrogen level is the highest at the late-proestrus stage, the results obtained from these mice were compared with those from ArKO mice. Specific stages of the estrus cycle were checked conventionally by photomicrographs of vaginal secretions (vaginal smear test; Supplementary Fig. 1A). ArKO mice showed a diestrus feature on the vaginal secretions that consisted predominantly of leukocytes as previously reported (Honda et al., 1998). In addition to the conventional vaginal smear test, regular estrus cycles were monitored in 24 wild-type mice by vaginal impedance measurements (MC-10C impedance checker; Muromachi, Tokyo, Japan) for 20-30 days (Maguire et al., 2005), and five late-proestrus mice obtained by this method
(Supplementary Fig. 1B) were included to 7 proestrus wild-type mice for ECG recording.

\section{RNA extraction and quantitative real-time reverse transcription polymerase chain reaction (RT-PCR)}

Total RNA was extracted from murine atria or ventricles using an RNeasy Mini Kit (Qiagen, Tokyo, Japan) and SYBR green real-time RT-PCR using an ABI7300 cycler (Applied Biosystems, Foster City, CA, USA). Gene expression was identified with appropriate primers (murine ether-a-go-go-related gene [mERG] sense primer, 5'-TCTGAACCTATATGCCCGTCCT-3'; mERG antisense primer, 5'-GCTGCTCTGTGTCCTTGTCTGT-3'; mSCN5A sense primer, 5'-AGAAGACGGTCCCAGAGCATTGAA-3'; mSCN 5 A antisense primer, 5'-ATGACCAGGAGCTGAGGTTCCAAA-3'; mCACNA1C sense primer, 5'-TGCTGTACTGGATGCAAGACGCTA-3' ; mCACNA1C antisense primer, 5'-GCATGCTCATGTTTCGAGGCTTGT-3'; mKCNJ2 sense primer, 5'-CCTTTGTAGTGCCAGAGACTTAG-3'; mKCNJ2 antisense primer, 5'-TCCTCTTCCTCTTTGCTTGTTAG-3'; mKCND3 sense primer, 5'-TGAACTGGCCTTCTATGGCATCCT-3'; mKCND3 antisense primer, 5'- TGATGACCGAGACGGCAATGAAGA-3') (Jeong et al., 2010). Transcript quantities were compared with those of the $\Delta \Delta \mathrm{Ct}$ method. Glyceraldehyde-3-phosphate dehydrogenase (GAPDH) was used as a housekeeping control (sense primer, 5' - ACCACAGTCCATGCCATCAC-3'; antisense primer, 5' - TCCACCACCCTGTTGCTGTA-3').

\section{ECG recordings}

The mice were deeply anesthetized by an intraperitoneal injection of urethane at $0.8 \mathrm{~g} / \mathrm{kg}$ body weight ( $\mathrm{g} / \mathrm{kg} \mathrm{BW}$ ), and all efforts were made to minimize suffering. Additional doses were given during the procedure as needed to maintain the anesthesia. All experiments were performed between 11:00 and 18:00. The murine trachea was cut down to guide the placement of an endotracheal tube. The mice were ventilated on room air $(0.5 \mathrm{~mL}$; 100-120 cycle/min) using a rodent ventilator (SN-480-7; Shinano Manufacturing Co., Tokyo, Japan). Body temperature was monitored with a rectal probe and maintained at $37^{\circ} \mathrm{C}$ by an incandescent lamp. ECG recordings were made using a telemetry transmitter (TA10ETAF20; Data Sciences, New Brighton, MN, USA) with subcutaneous leads placed in the conventional lead II position (Kuma et al., 2004). A jugular vein was cannulated for drug administration. The drug injection rate was controlled using a micrometer syringe injec- 
Effects of endogenous estrogen on murine ECG

A

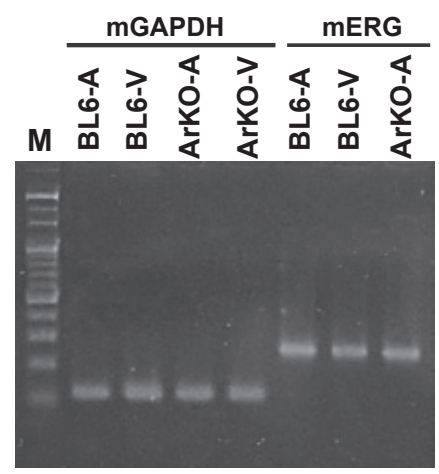

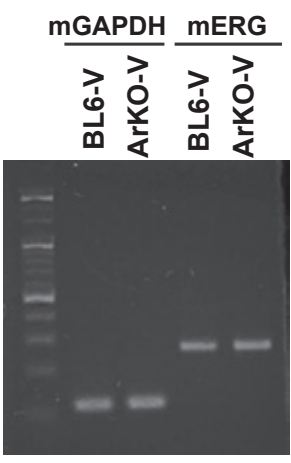

B
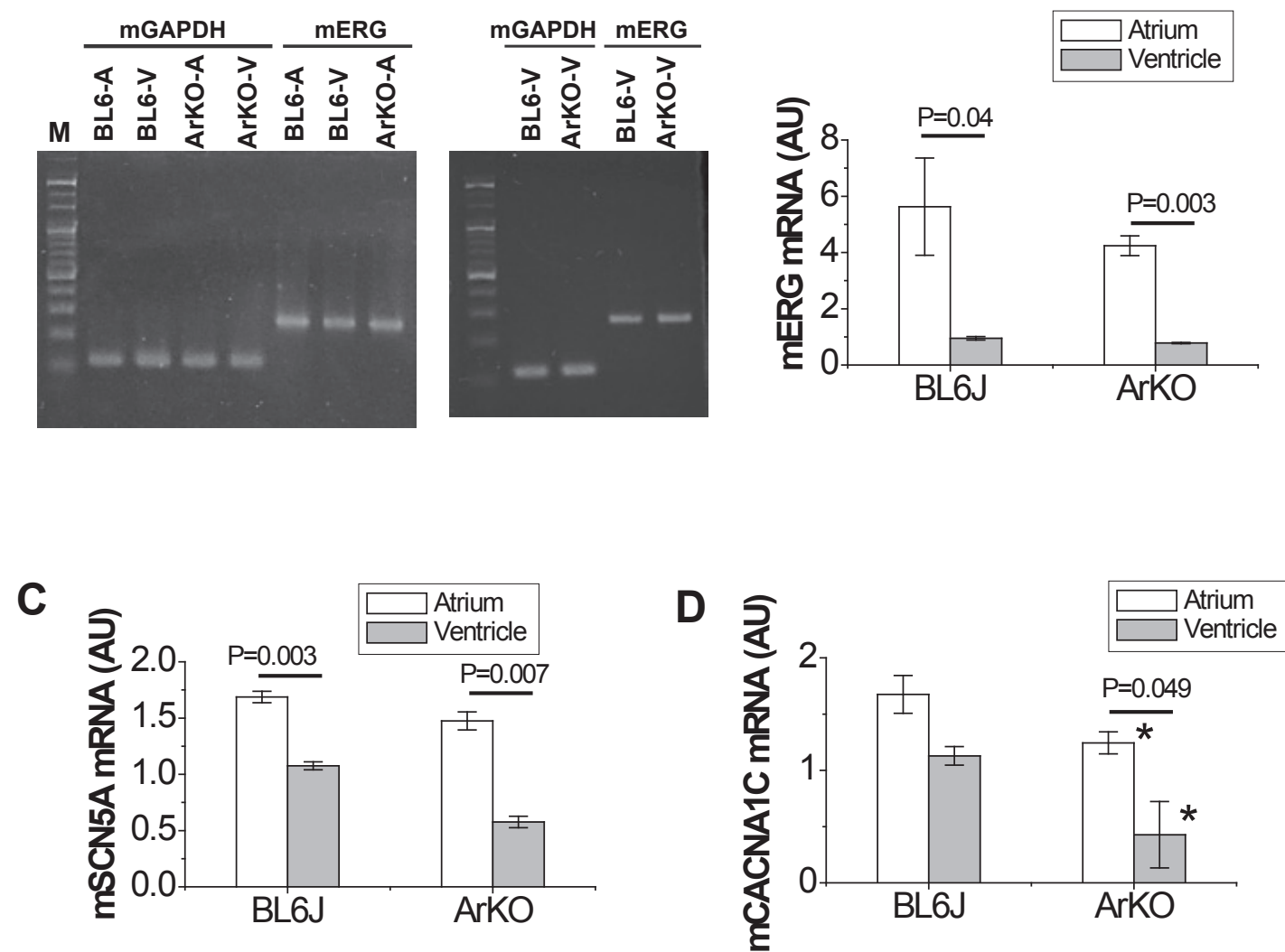

E

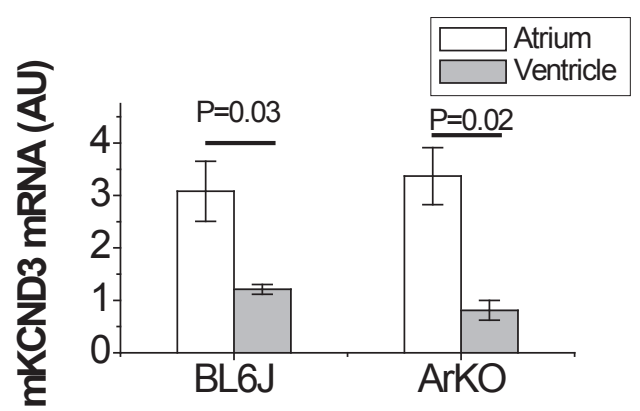

$\mathbf{F}$

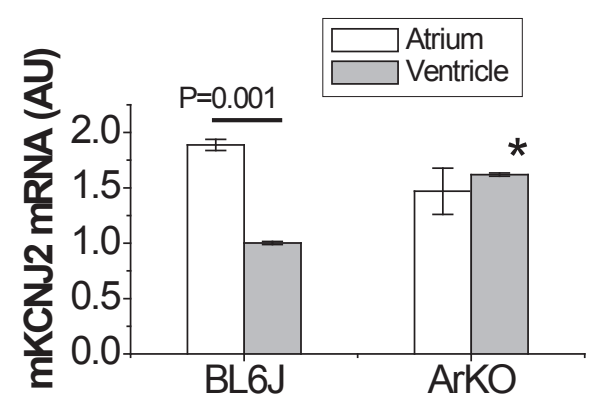

Fig. 1. Analysis of murine ERG mRNA in the murine atrium and ventricle. (A) Detection of mERG mRNA in the murine heart using reverse transcription polymerase chain reaction (RT-PCR). Total RNA from the atria and ventricles was reverse transcribed and the cDNA was subjected to PCR. The photograph shows representative murine ERG or glyceraldehyde-3-phosphate dehydrogenase (GAPDH) RT-PCR products after electrophoresis on a 3\% agarose gel containing ethidium bromide from the following targets: atrium of wild-type (C57/BL6J) mice (lane BL6-A), ventricle of wild-type mice (lane BL6-V), atrium of aromatase cytochrome P450 knockout (ArKO) heterozygote mice (lane ArKO-A), and ventricle of ArKO heterozygote mice (lane ArKO-V). Lane M: 100-bp DNA ladder marker of Bio-Regenerations-Japan (Cat\#BRG-100-02). (B-F) Ion channel mRNA levels were determined by reverse transcription and quantitative PCR (real time RT-PCR) and normalized to GAPDH mRNA. Quantification of mRNA in the atria and ventricles obtained from proestrus female BL6J (wildtype) mice and ArKO heterozygous female mice. $\mathrm{N}=3$ for the PCR reaction. The data are expressed as mean and S.E.M. of three independent experiments. (B) mERG, $\mathrm{I}_{\mathrm{Kr}}$ (C) mSCN5A, Nav1.5. (D) mCACNA1C, Cav1.2. (E) mKCND3, Kv4.3. (F) mKCNJ2, Kir2.1. Statistical significance in expression levels was analyzed using an unpaired $t$-test $(P$ values in the graphs, atria vs. ventricle, ${ }^{*} P<0.05$, wild-type vs. ArKO). 
tor $(0.04 \mathrm{~mL} / \mathrm{min})$, and the injection volume was $2 \mathrm{~mL} / \mathrm{kg} \mathrm{BW}$. E-4031 administration was started after heart rate (HR) was stabilized with autonomic blockade by sequential intravenous injections of atropine $(0.5 \mathrm{mg} / \mathrm{kg} \mathrm{BW})$ and propranolol (1 mg/kg BW). Data were acquired using PowerLab and Chart4 (version 4.2; ADI Instruments, Nagoya, Japan). The ECG recordings were measured as previously described (Berul et al., 1996) and the analyzed as described below.

\section{Chemicals}

The E-4031 was a gift from Esai Co. Ltd. (Tokyo, Japan). All other materials were of reagent grade obtained from standard sources. A stock E-4031 solution at $10 \mathrm{mM}$ $\left(\mathrm{H}_{2} \mathrm{O}\right)$ was diluted in saline to final concentrations.

\section{ECG Analysis}

ECG tracings were analyzed individually offline by three investigators blinded to the genotypes. LabChart4 or 7Pro software (ADI Instruments) using the ECG module were used for the data analysis. ECG waveforms were averaged by alignment at the peak of the $\mathrm{R}$ wave every four beats. The PR interval was measured from the beginning of the P wave to the onset of the QRS complex. The QT interval was measured from the onset of the QRS complex to the end of the T wave. The onset of the QRS complex was defined as the first deflection of the QRS complex, while the end of the $T$ wave was defined as the intersection of the descending part of the positive $\mathrm{T}$ wave with the isoelectric line. The QT intervals were corrected for HR using a modified Bazzet formula, $\mathrm{QT}_{\mathrm{C}}$ $=\mathrm{QT} /(\mathrm{RR} / 100)^{1 / 2}($ Knollmann et al., 2001), with QT and RR expressed in milliseconds. HR was calculated by the equation $\mathrm{HR}(\mathrm{BPM})=60000 / \mathrm{RR}(\mathrm{ms})$.

\section{Other data analysis}

All values are presented as mean \pm S.E.M. Graphical and statistical analyses were carried out using Origin 7.0J/9.1 J software (OriginLab, Northampton, MA, USA) and InStat program (Graphpad, La Jolla, CA, USA). Unless otherwise noted, statistical significance was assessed using the Mann-Whitney $U$ test for simple comparisons, and the Friedman test followed by the Dunn test for multiple comparisons. Differences at $P<0.05$ were considered significant.

\section{RESULTS}

To test whether estrogens influence the effects of $\mathrm{I}_{\mathrm{Kr}}$ channel blockers on cardiac electrophysiology, we compared the effect of the selective $\mathrm{I}_{\mathrm{Kr}}$ channel blocker E-4031 on ECG parameters between wild-type and ArKO female mice. First, mERG expression was studied by real time RT-PCR in wild-type and ArKO heterozygote murine hearts (Fig. 1). Fig. 1A shows that each reaction efficiently amplified a single product to ensure accurate template quantification on real time RT-PCR. As shown in Fig. 1B, mERG mRNA expression in the atrium was significantly higher than that in the ventricle, while the $\mathrm{mERG}$ expression levels in the same region of the hearts were equivalent regardless of mouse type. When other ion channel expression was compared among the mouse line types (Figs. 1C-F), the ArKO mouse line showed lower CACNA1C expression in atria and ventricle and higher KCNJ2 expression in ventricle, although the baseline ECG were very similar (Table 1). The heterogeneity of

Table 1. Baseline of murine ECG parameters.

\begin{tabular}{llcc}
\hline & & Non-treatment & Atropine + propranolol \\
\hline & HR (BPM) & $595.1 \pm 30.3$ & $529.0 \pm 18.2$ \\
Wild type (proestrus) & PR (ms) & $35.2 \pm 1.4$ & $36.0 \pm 1.3$ \\
$(\mathrm{n}=7)$ & QT (ms) & $19.7 \pm 0.6$ & $20.7 \pm 0.7$ \\
& $\mathrm{QT}_{\mathrm{C}}(\mathrm{ms})$ & $19.5 \pm 0.6$ & $19.5 \pm 0.6$ \\
\hline & HR $(\mathrm{BPM})$ & $618.0 \pm 15.0$ & $526.6 \pm 10.4^{* * *}$ \\
ArKO & PR (ms) & $35.2 \pm 1.0$ & $35.2 \pm 0.8$ \\
$(\mathrm{n}=9)$ & QT (ms) & $20.8 \pm 0.6$ & $22.4 \pm 1.1$ \\
& $\mathrm{QT}_{\mathrm{C}}(\mathrm{ms})$ & $21.2 \pm 0.8$ & $21.0 \pm 1.0$ \\
\hline
\end{tabular}

Lead II ECG parameters (RR, PR, QT, and QTc intervals) were recorded from wild type $(\mathrm{n}=7)$ and ArKO $(\mathrm{n}=9)$ female mice. Pharmacological denervation was performed administration of atropine $(0.5 \mathrm{mg} / \mathrm{kg} \mathrm{BW})$ and propranolol $(1 \mathrm{mg} / \mathrm{kg} \mathrm{BW})$. The wild type female mice at proestrus stage identified by smear test were used for ECG measurements. In the wild type mice, 5 out of 7 were determined at the proestrus state both with smear test and vaginal impedance measurement (see detail in the methods).

$* * *, p<0.001$ : vs non-treatment, Wilcoxon signed-rank test. 
Effects of endogenous estrogen on murine ECG

mERG expression in Fig. 1B is consistent with the functional $I_{\mathrm{Kr}}$ expression in adult murine ventricular myocytes being rather low (Trépanier-Boulay et al., 2004), but $I_{\mathrm{Kr}}$ expressions in the sinoatrial and atrioventricular (A-V) node are high enough to contribute to pacemaker activity of these cells (Marger et al., 2011), implying that $\mathrm{I}_{\mathrm{Kr}}$ blockade alters normal electrical function from sinoatrial to A-V node. Thus, to examine in vivo impacts of murine $I_{\mathrm{Kr}}$ modulation, $\mathrm{HR}$ and $\mathrm{A}-\mathrm{V}$ nodal conduction times (PR intervals) were intensively studied on surface ECG. QT intervals were also studied as references because the mERG expression in the ventricle was detected even if it was at a lower level than that in the atrium.

Because we reported that $I_{\mathrm{Kr}}$ suppression extent by estrogen was moderate (10-20\%) (Kurokawa et al., 2008), it can be predicted that in vivo consequences may be moderate as well. To detect moderate changes in ECG parameters, it is necessary to reduce variations in baseline ECG. It is known that autonomic regulation increases variations in baseline ECG parameters such as HR and QT intervals (Sasano et al., 2004). Thus, a pharmacological denervation was performed by the administration of atropine $(0.5 \mathrm{mg} / \mathrm{kg} \mathrm{BW})$ and propranolol (1 mg/ $\mathrm{kg} \mathrm{BW})$ prior to the drug testing.

Table 1 shows the HR, PR intervals, QT intervals, and $\mathrm{QT}_{\mathrm{C}}$ intervals measured from the surface ECG in wildtype and ArKO mice; however, no significant difference in ECG parameters was seen between them. Denervation significantly reduced the HR of ArKO mice. Although a denervation-induced reduction in HR was also seen in the wild-type mice, the effect was not statistically significant, which may have been influenced, at least in part, by a difference in variation before and after denervation. This dif- ference was evidenced by the greater standard deviation of HR in the wild-type mice (74.2 ms) compared to that in ArKO mice (42.5 ms). Consistent with former studies (Sasano et al., 2004), the denervation did not affect ratecorrected QT $\left(\mathrm{QT}_{\mathrm{C}}\right)$ intervals. Importantly, the ECG values recorded with the denervation were similar to those of wild-type versus ArKO mice, providing good baseline values for future pharmacological investigations.

Figure 2 shows that E-4031 was cumulatively administrated under pharmacological denervation by intravenous injection $0.1-10 \mathrm{mg} / \mathrm{kg} \mathrm{BW}$. The time courses of $\mathrm{HR}, \mathrm{PR}$ intervals, QT intervals, and $\mathrm{QT}_{\mathrm{C}}$ intervals during E-4031 administration were monitored in wild-type and ArKO mice (Figs. 2C-F). In both mice, E-4031 reduced HR in a dose-dependent manner (Fig. 2C, Table 2; Friedman test). E-4031-induced bradycardia occurred $<5$ min after intravenous administration and was sustained for at least $15 \mathrm{~min}$ at 0.1 and $1 \mathrm{mg} / \mathrm{kg} \mathrm{BW} \mathrm{E-4031.} \mathrm{When}$ E-4031 (10 mg/kg BW) was administered, transient bradycardia occurred followed by sustained bradycardia in the late-proestrus wild-type and ArKO mice (Fig. 2C). Figure 3A shows that aromatase knockout in ArKO mice significantly reduced the dose-dependent bradycardia extent in the late-proestrus mice after a $0.1 \mathrm{mg} / \mathrm{kg} \mathrm{BW}$ injection of E-4031 (Mann-Whitney test, $P<0.05$; proestrus vs. ArKO).

Figure 2D and Table 2 show that the PR intervals in wild-type mice were slightly but significantly prolonged by E-4031 (10 mg/kg BW), suggesting a contribution of $I_{\mathrm{Kr}}$ to A-V conducting velocity. There was no significant effect of E-4031 (10 mg/kg BW) on PR intervals in ArKO mice (Fig. 3B, Table 2), suggesting a sharp contrast to the effects of E-4031 on A-V conducting velocity

Table 2. Effects of E-4031 on murine ECG parameters.

\begin{tabular}{llcccc}
\hline & & Control & $0.1 \mathrm{mg} / \mathrm{kgBW}$ & $1 \mathrm{mg} / \mathrm{kgBW}$ & $\begin{array}{c}10 \mathrm{mg} / \mathrm{kgBW} \\
\mathrm{E}-4031\end{array}$ \\
\hline & & E-4031 & E-4031 & $464.8 \pm 13.7^{* * *}$ \\
Proestrus & HR (BPM) & $529.0 \pm 18.2$ & $501.5 \pm 18.8$ & $490.2 \pm 15.8^{*}$ & $38.9 \pm 2.3^{* *}$ \\
& PR (ms) & $36.0 \pm 1.3$ & $36.4 \pm 1.3$ & $36.6 \pm 1.5$ & $27.1 \pm 1.4^{* * *}$ \\
& QT (ms) & $20.7 \pm 0.7$ & $21.2 \pm 0.9$ & $21.9 \pm 0.8$ & $23.7 \pm 1.2^{*}$ \\
\hline ArKO & QT $(\mathrm{ms})$ & $19.5 \pm 0.6$ & $19.4 \pm 0.6$ & $20.1 \pm 0.8$ & $484.4 \pm 8.1^{*}$ \\
$(\mathrm{n}=9)$ & HR (BPM) & $526.6 \pm 10.4$ & $518.2 \pm 9.8$ & $514.8 \pm 8.2$ & $35.0 \pm 1.0$ \\
& PR (ms) & $35.2 \pm 0.8$ & $35.4 \pm 0.8$ & $34.4 \pm 0.7$ & $24.9 \pm 1.3^{*}$ \\
& QT (ms) & $22.4 \pm 1.1$ & $22.3 \pm 1.1$ & $22.6 \pm 0.8$ & $22.3 \pm 1.0$ \\
\hline
\end{tabular}

Lead II ECG parameters in proestrus wild type $(\mathrm{n}=7$, including seven proestrus mice) and ArKO $(\mathrm{n}=9)$ female mice pretreated with atropine $(0.5 \mathrm{mg} / \mathrm{kg} \mathrm{BW})$ and propranolol $(1 \mathrm{mg} / \mathrm{kg} \mathrm{BW})$ before (control) and after cumulative injection of E-4031 every $10 \mathrm{minutes}$. ${ }^{*}, * *, * * *, p<0.05, p<0.01$, and $p<0.001$, respectively, $v s$ control, Friedman test. 
J. Kurokawa et al.

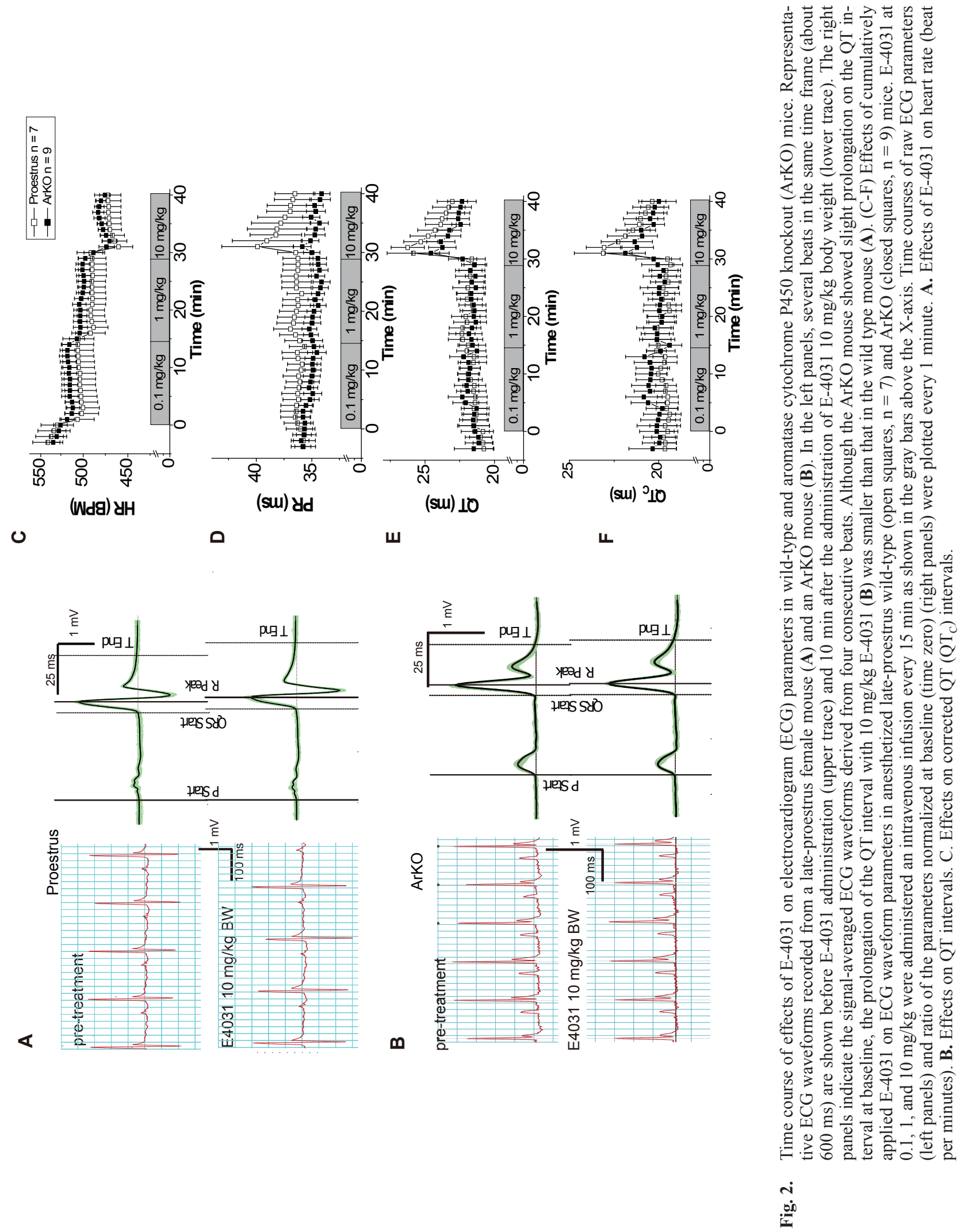


Effects of endogenous estrogen on murine ECG

A

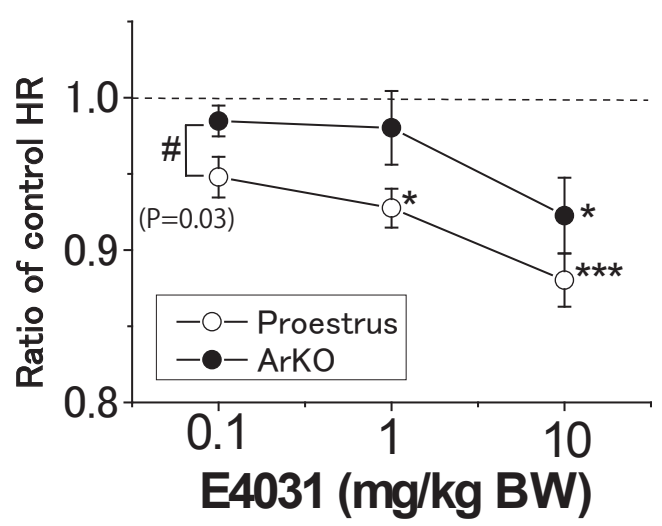

C

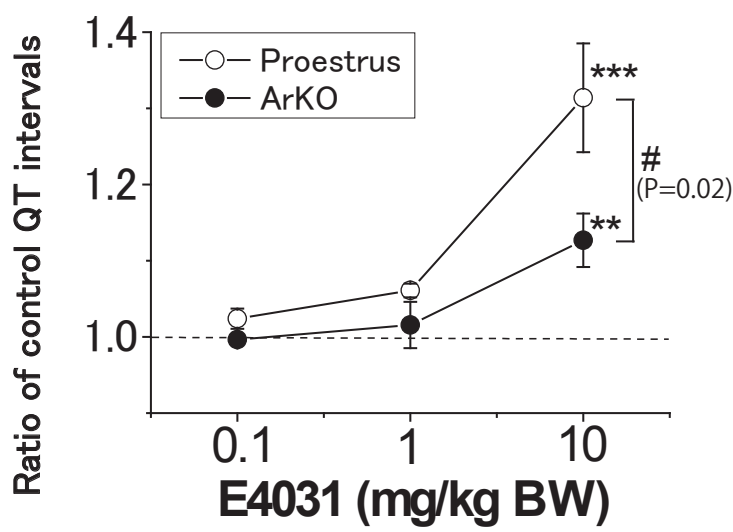

B

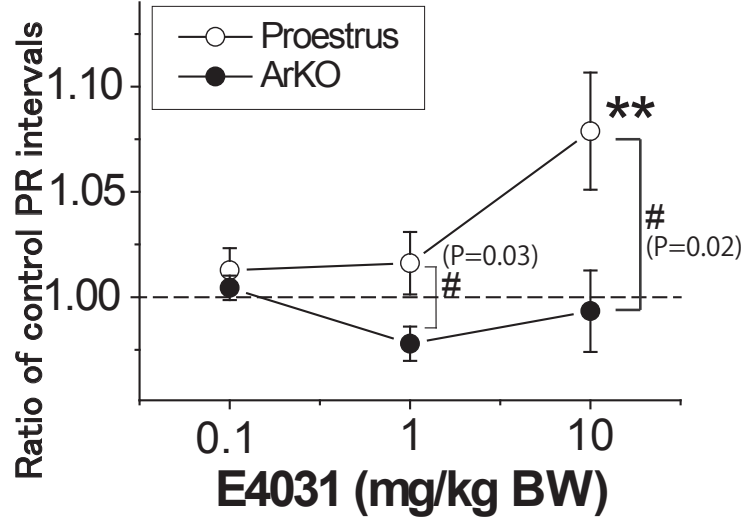

D

Fig. 3. Dose-dependent changes in electrocardiography (ECG) parameters by E-4031 administration in late-proestrus wild-type and aromatase cytochrome P450 knockout (ArKO) mice. The relative changes of heart rate (A), QT interval (B), and corrected QT $\left(\mathrm{QT}_{\mathrm{C}}\right)$ interval $(\mathbf{C})$ in response to the cumulative administration of E-4031 $(0.1,1$, and $10 \mathrm{mg} / \mathrm{kg})$ compared between late-proestrus wild-type mice (proestrus; open circles) and ArKO mice (closed circles). Maximum extent of parameters at each dose were determined by analyzing averaged ECG waveforms (four beats) and plotted against the dosage. All parameters are expressed as relative value normalized by baseline (time zero) levels. Statistical significance between proestrus and ArKO mice was analyzed using Mann-Whitney test $(\# P<0.05)$. Statistical significance in effects of E-4031 was analyzed using the Friedman test followed by Dunn's test for multiple comparisons $(* P<0.05, * * P<0.01, * * * P<0.001$ vs. control).

in wild-type mice.

E-4031 prolonged QT and $\mathrm{QT}_{\mathrm{C}}$ intervals in wild-type mice (Figs. 2E and 2F, Fig. 3, Table 2). In the late-proestrus female wild-type mice, E-4031 significantly prolonged QT and $\mathrm{QT}_{\mathrm{C}}$ intervals at $10 \mathrm{mg} / \mathrm{kg} \mathrm{BW}$ (Friedman test, $P<0.05$ vs. control). In the ArKO mice, E-4031 at $10 \mathrm{mg} / \mathrm{kg}$ BW significantly prolonged QT intervals (Friedman test, $P<0.05$ vs. control), but the extent of the E-4031-induced QT prolongation was reduced
(Fig. 3C) and did not prolong $\mathrm{QT}_{\mathrm{C}}$ interval. Representative ECG waveforms (Figs. 2A, 2B right panels) show that the extent of E-4031 prolongation in the ArKO mice was smaller than that in the wild-type mice. Indeed, the extent of the QT prolongation at $10 \mathrm{mg} / \mathrm{kg} \mathrm{BW} \mathrm{E-4031}$ in the ArKO mice was significantly less than that in the late-proestrus wild-type mice (Fig. 3; Mann-Whitney test, $P<0.05$; wild-type vs. ArKO). These results indicate that QT and $\mathrm{QT}_{\mathrm{C}}$ intervals in ArKO mice have lower sensitiv- 
ity to E-4031.

\section{DISCUSSION}

We have shown here that the physiological levels of estradiol and estron sulfate directly downregulate the hERG channel and increase E-4031-induced hERG modulation in in vitro and ex vivo conditions (Kakusaka et al., 2009; Kurokawa et al., 2008). In this study, we investigated the influence of endogenous estrogen in effects of a hERG blocker on cardiac electrophysiology by employing aromatase knockout mice. Analysis of the ECG recordings revealed that ablation of the endogenous estrogens significantly blunted the effects of E-4031 on HR and QT intervals in mice under a denervation condition, suggesting that aromatic centroid of estrogens increased E-4031 sensitivity. Thus, this could be the first report to present evidence that endogenous estrogens increase the sensitivity of a hERG blocker in the in vivo condition.

Female sex is an independent risk factor for developing drug-induced torsades de pointes (TdP). This is often attributed to longer $\mathrm{QT}_{\mathrm{C}}$ intervals in women than in men. The sex difference is thought to be caused by shortening of the action potential by endogenous testosterone and progesterone, which is supported by many clinical and basic studies (Kurokawa et al., 2012, 2009; Zhang et al., 2011; Charbit et al., 2009; Pecori Giraldi et al., 2010). Instead, estrogen has both antiarrhythmic and arrhythmic effects. The vasodilation effects of estrogen indirectly mask arrhythmic risks by protecting against cardiac ischemia-reperfusion injury (Murphy and Steenbergen, 2008). It has been reported in animal models that estrogen has a profound impact in some drug-induced long QT syndrome mainly by reducing repolarization reserve (Hara et al., 1998; Drici et al., 1998; Kurokawa et al., 2009; Saito et al., 2009). This study's findings clearly support that estrogen predisposes women to the development of druginduced arrhythmias by lengthening the QT interval. However, the clinical impact of endogenous estrogen on ECG parameters has yet to be clarified. Our data consistently showed no significant effects of ArKO in the baseline values (Fig. 2). Although conflicting results exist regarding the effects of endogenous estrogen on the cardiac repolarization process, several clinical studies have reported that exogenous estrogen therapy but not progesterone/estrogen therapy lengthens the QT intervals (Kadish et al., 2004; Carnethon et al., 2003; Larsen et al., 1998). However, the transcriptional targets of estrogen have not been clarified, and no studies have examined the delivery route of exogenous estrogens. Thus, further studies are needed to clarify the clinical impact of endogenous and exogenous estrogens.

In this study, the usage of the ArKO mice (Honda et al., 1998) allows us to address the effect of complete lack of estrogen on the heart (Haines et al., 2012), because other murine models of estrogen privation such as ovariectomy (Thum and Borlak, 2002) or global estrogen receptor (ER) deletions (Rissman et al., 1997; Skavdahl et al., 2005) cannot abrogate estrogen action. Although both ER $\alpha \mathrm{KO}$ and the ER $\beta K O$ mice show significant hypertension and systemic hypoxia (Morani et al., 2006; Rissman et al., 1997), these phenotypes are largely explained by ligand-independent actions (Qin et al., 2008). To our knowledge, this is the first report to study cardiac electrophysiology in ArKO mice.

Small rodents such as mice show different regional heterogeneity of ion channel expression in the heart from larger mammals (Panama et al., 2007). Thus, it is important to consider the regional heterogeneity of ion channel expression of the mouse heart. Because $\mathrm{mERG}$ expression is higher in murine atrium, we rather focused on measurements of HR (RR intervals) and PR intervals, which can be easily measured by discerning peak of $R$ wave and start of $\mathrm{P}$ wave. Unlike larger animals including humans, the use of murine ECG makes it difficult to discern the end of QRS and the onset and end of the T wave due to the lack of a plateau of action potential (Knollmann et al., 2001; Liu et al., 2004). Thus, in this study, the end of the $\mathrm{T}$ wave was checked by four individuals as blind tests but resulted in the same significant effects. Bradycardia induced by E- 4031 was detectable from $0.1 \mathrm{mg} / \mathrm{kg} \mathrm{BW}$, where QT intervals had no effect (Fig. 2 and Table 2). It was difficult to detect $\mathrm{QT}_{\mathrm{C}}$ prolongation by E-4031 in wild-type FVB mice (Lande et al., 2001), which is a different strain from the one in this study. In the same group, we detected the effects of E-4031 on HR that is consistent with our data, showing a contribution of the $\mathrm{I}_{\mathrm{Kr}}$ channel in sinoatrial node (Lande et al., 2001). Therefore, the effects on HR is also caused by inhibition of the $\mathrm{I}_{\mathrm{Kr}}$ currents because they are functionally expressed in murine sinoatrial myocytes (Cho et al., 2003). Although E-4031 $10 \mathrm{mg} / \mathrm{kg}$ BW showed strong bradycardia (Table 2), aromatase knockout significantly decreased E-4031-induced $\mathrm{QT}_{\mathrm{C}}$ prolongation, indicating that ablation of endogenous estrogen affects E-4031 sensitivity. Recent data depict the importance of aromatase expression in the heart, which shows protective effects on ischemic myocardial cells and coronary vasculature damage (Bell et al., 2014; Jazbutyte et al., 2012).

In Fig. 1, in the ArKO mice compared with wild-type, the expression of CACNA1C (Cav1.2) was lower whereas that of KCNJ2 (Kir2.1) was higher. Although it is dif- 
Effects of endogenous estrogen on murine ECG

ficult to predict how these changes affect cardiac action potential, the basal QT and $\mathrm{QT}_{\mathrm{C}}$ intervals were very similar between ArKO and wild-type mice (Table 1). Nevertheless, it is possible that these changes in channel expression might alter the sensitivity of ECG parameters to E-4031. Thus, this is the limitation of this study.

In conclusions, the electrophysiological and pharmacological analyses in this study provide important information about aromatase function for medical application. In sum, our results provide an in vivo proof of principle and demonstrate that endogenous estrogens increase E-4031 sensitivity for inhibiting murine $\mathrm{I}_{\mathrm{Kr}}$ currents.

\section{ACKNOWLEDGMENTS}

We thank Mr. Masaji Tamagawa (Chiba University) for recording the ECG, and Ms. T. Ando, Ms. Sayuri Goryoda, and Dr. H. Onishi (TMDU, MRI) for performing quality checks of the ECG analyses, and Mr. K Takahashi for helping RT-PCR.

This work was supported by a grant-in-aid for scientific research on priority areas (23136703 to JK) and grants (25461045 to TS, 23390205 and 25670126 to $\mathrm{TF}, 15 \mathrm{H} 04684$ to JK, 23390053 to $\mathrm{HN}$ ) from the Ministry of Education, Science, Culture, Sports and Technology of Japan (www.mext.go.jp/), Restart Postdoctoral Fellowship from Japan Society for the Promotion of Science (JSPS to MK) and Takeda Science Foundation (to JK, URL; www.takeda-sci.or.jp/). This work was also supported by a program of Activation of Gender Equity/Gender Medicine and Enrichment of Life of Tokyo Medical and Dental University (to JK and MK).

Conflict of interest---- The authors declare that there is no conflict of interest.

\section{REFERENCES}

Bell, J.R., Bernasochi, G.B., Varma, U., Boon, W.C., Ellem, S.J., Risbridger, G.P. and Delbridge, L.M. (2014): Aromatase transgenic upregulation modulates basal cardiac performance and the response to ischemic stress in male mice. Am. J. Physiol. Heart Circ. Physiol., 306, H1265-74.

Berul, C.I., Aronovitz, M.J., Wang, P.J. and Mendelsohn, M.E. (1996): In vivo cardiac electrophysiology studies in the mouse. Circulation, 94, 2641-2648.

Carnethon, M.R., Anthony, M.S., Cascio, W.E., Folsom, A.R., Rautaharju, P.M., Liao, D., Evans, G.W. and Heiss, G. (2003): A prospective evaluation of the risk of QT prolongation with hormone replacement therapy: the atherosclerosis risk in communities study. Ann. Epidemiol., 13, 530-536.

Charbit, B., Christin-Maître, S., Démolis, J.L., Soustre, E., Young,
J. and Funck-Brentano, C. (2009): Effects of testosterone on ventricular repolarization in hypogonadic men. Am. J. Cardiol., 103, 887-890.

Cho, H.S., Takano, M. and Noma, A. (2003): The electrophysiological properties of spontaneously beating pacemaker cells isolated from mouse sinoatrial node. J. Physiol., 550, 169-180.

Drici, M.D., Knollmann, B.C., Wang, W.X. and Woosley, R.L. (1998): Cardiac actions of erythromycin: influence of female sex. JAMA, 280, 1774-1776.

Haines, C.D., Harvey, P.A. and Leinwand, L.A. (2012): Estrogens mediate cardiac hypertrophy in a stimulus-dependent manner. Endocrinology, 153, 4480-4490.

Hara, M., Danilo, P.Jr. and Rosen, M.R. (1998): Effects of gonadal steroids on ventricular repolarization and on the response to E4031. J. Pharmacol. Exp. Ther., 285, 1068-1072.

Honda, S., Harada, N., Ito, S., Takagi, Y. and Maeda, S. (1998): Disruption of sexual behavior in male aromatase-deficient mice lacking exons 1 and 2 of the cyp 19 gene. Biochem. Biophys. Res. Commun., 252, 445-449.

Jazbutyte, V., Stumpner, J., Redel, A., Lorenzen, J.M., Roewer, N., Thum, T. and Kehl, F. (2012): Aromatase inhibition attenuates desflurane-induced preconditioning against acute myocardial infarction in male mouse heart in vivo. PloS One, 7, e42032.

Jeong, J.H., Jung, Y.K., Kim, H.J., Jin, J.S., Kim, H.N., Kang, S.M., Kim, S.Y., van Wijnen, A.J., Stein, J.L., Lian, J.B., Stein, G.S., Kato, S. and Choi, J.Y. (2010): The gene for aromatase, a ratelimiting enzyme for local estrogen biosynthesis, is a downstream target gene of Runx2 in skeletal tissues. Mol. Cell. Biol., 30, 2365-2375.

Kadish, A.H., Greenland, P., Limacher, M.C., Frishman, W.H., Daugherty, S.A. and Schwartz, J.B. (2004): Estrogen and progestin use and the QT interval in postmenopausal women. Ann. Noninvasive Electrocardiol., 9, 366-374.

Kakusaka, S., Asayama, M., Kaihara, A., Sasano, T., Suzuki, T., Kurokawa, J. and Furukawa, T. (2009): A receptor-independent effect of estrone sulfate on the HERG channel. J. Pharmacol. Sci., 109, 152-156.

Knollmann, B.C., Katchman, A.N. and Franz, M.R. (2001): Monophasic action potential recordings from intact mouse heart: validation, regional heterogeneity, and relation to refractoriness. J. Cardiovasc. Electrophysiol., 12, 1286-1294.

Kuma, A., Hatano, M., Matsui, M., Yamamoto, A., Nakaya, H., Yoshimori, T., Ohsumi, Y., Tokuhisa, T. and Mizushima, N. (2004): The role of autophagy during the early neonatal starvation period. Nature, 432, 1032-1036.

Kurokawa, J. and Furukawa, T. (2013): Non-genomic action of sex steroid hormones and cardiac repolarization. Biol. Pharm. Bull., 36, 8-12.

Kurokawa, J., Kodama, M., Furukawa, T. and Clancy, C.E. (2012): Sex and gender aspects in antiarrhythmic therapy. Handb. Exp. Pharmacol., 214, 237-263.

Kurokawa, J., Suzuki, T. and Furukawa, T. (2009): New aspects for the treatment of cardiac diseases based on the diversity of functional controls on cardiac muscles: acute effects of female hormones on cardiac ion channels and cardiac repolarization. J. Pharmacol. Sci., 109, 334-340.

Kurokawa, J., Tamagawa, M., Harada, N., Honda, S., Bai, C.X., Nakaya, H. and Furukawa, T. (2008): Acute effects of oestrogen on the guinea pig and human IKr channels and drug-induced prolongation of cardiac repolarization. J. Physiol., 586, 29612973.

Lande, G., Demolombe, S., Bammert, A., Moorman, A., Charpentier, 
F. and Escande, D. (2001): Transgenic mice overexpressing human KvLQT1 dominant-negative isoform. Part II: Pharmacological profile. Cardiovasc. Res., 50, 328-334.

Larsen, J.A., Tung, R.H., Sadananda, R., Goldberger, J.J., Horvath, G., Parker, M.A. and Kadish, A.H. (1998): Effects of hormone replacement therapy on QT interval. Am. J. Cardiol., 82, 993995.

Liu, G., Iden, J.B., Kovithavongs, K., Gulamhusein, R., Duff, H.J. and Kavanagh, K.M. (2004): In vivo temporal and spatial distribution of depolarization and repolarization and the illusive murine T wave. J. Physiol., 555, 267-279.

Maguire, J.L., Stell, B.M., Rafizadeh, M. and Mody, I. (2005): Ovarian cycle-linked changes in GABA(A) receptors mediating tonic inhibition alter seizure susceptibility and anxiety. Nat. Neurosci., 8, 797-804.

Makkar, R.R., Fromm, B.S., Steinman, R.T., Meissner, M.D. and Lehmann, M.H. (1993): Female gender as a risk factor for torsades de pointes associated with cardiovascular drugs. JAMA, 270, 2590-2597.

Marger, L., Mesirca, P., Alig, J., Torrente, A., Dubel, S., Engeland, B., Kanani, S., Fontanaud, P., Striessnig, J., Shin, H.S., Isbrandt, D., Ehmke, H., Nargeot, J. and Mangoni, M.E. (2011): Pacemaker activity and ionic currents in mouse atrioventricular node cells. Channels (Austin), 5, 241-250.

Miki, T., Suzuki, M., Shibasaki, T., Uemura, H., Sato, T., Yamaguchi, K., Koseki, H., Iwanaga, T., Nakaya, H. and Seino, S. (2002): Mouse model of Prinzmetal angina by disruption of the inward rectifier Kir6.1. Nat. Med., 8, 466-472.

Morani, A., Barros, R.P., Imamov, O., Hultenby, K., Arner, A., Warner, M. and Gustafsson, J.A. (2006): Lung dysfunction causes systemic hypoxia in estrogen receptor beta knockout (ERbeta-/-) mice. Proc. Natl. Acad. Sci. USA, 103, 7165-7169.

Murphy, E. and Steenbergen, C. (2008): Mechanisms underlying acute protection from cardiac ischemia-reperfusion injury. Physiol. Rev., 88, 581-609.

Panama, B.K., McLerie, M. and Lopatin, A.N. (2007): Heterogeneity of IK1 in the mouse heart. Am. J. Physiol. Heart Circ. Physiol., 293, H3558-3567.

Pecori Giraldi, F., Toja, P.M., Filippini, B., Michailidis, J., Scacchi, M., Stramba Badiale, M. and Cavagnini, F. (2010): Increased prevalence of prolonged QT interval in males with primary or secondary hypogonadism: a pilot study. Int. J. Androl., 33, e132138.

Qin, X., Wang, X.H., Yang, Z.H., Ding, L.H., Xu, X.J., Cheng, L., Niu, C., Sun, H.W., Zhang, H. and Ye, Q.N. (2008): Repression of NFAT3 transcriptional activity by estrogen receptors. Cellular and molecular life sciences : CMLS, 65, 2752-2762.

Rissman, E.F., Wersinger, S.R., Taylor, J.A. and Lubahn, D.B. (1997): Estrogen receptor function as revealed by knockout studies: neuroendocrine and behavioral aspects. Horm. Behav., 31, 232-243.

Rodriguez, I., Kilborn, M.J., Liu, X.K., Pezzullo, J.C. and Woosley, R.L. (2001): Drug-induced QT prolongation in women during the menstrual cycle. Jama, 285, 1322-1326.

Saito, T., Ciobotaru, A., Bopassa, J.C., Toro, L., Stefani, E. and Eghbali, M. (2009): Estrogen contributes to gender differences in mouse ventricular repolarization. Circ. Res., 105, 343-352.

Sasano, T., Okishige, K., Azegami, K. and Isobe, M. (2004): Clinical assessment of antiarrhythmic agents for paroxysmal atrial fibrillation guided by modification of electrophysiologic arrhythmogenicity. J. Cardiovasc. Electrophysiol., 15, 1250-1257.

Skavdahl, M., Steenbergen, C., Clark, J., Myers, P., Demianenko, T., Mao, L., Rockman, H.A., Korach, K.S. and Murphy, E. (2005): Estrogen receptor-beta mediates male-female differences in the development of pressure overload hypertrophy. Am. J. Physiol. Heart Circ. Physiol., 288, H469-476.

Suzuki, M., Sasaki, N., Miki, T., Sakamoto, N., Ohmoto-Sekine, Y., Tamagawa, M., Seino, S., Marbán, E. and Nakaya, H. (2002): Role of sarcolemmal K(ATP) channels in cardioprotection against ischemia/reperfusion injury in mice. J. Clin. Invest., 109, 509-516.

Thum, T. and Borlak, J. (2002): Testosterone, cytochrome P450, and cardiac hypertrophy. FASEB J., 16, 1537-1549.

Trépanier-Boulay, V., Lupien, M.A., St-Michel, C. and Fiset, C. (2004): Postnatal development of atrial repolarization in the mouse. Cardiovasc. Res., 64, 84-93.

Zhang, Y., Ouyang, P., Post, W.S., Dalal, D., Vaidya, D., Blasco-Colmenares, E., Soliman, E.Z., Tomaselli, G.F., and Guallar, E. (2011): Sex-steroid hormones and electrocardiographic QT-interval duration: findings from the third National Health and Nutrition Examination Survey and the Multi-Ethnic Study of Atherosclerosis. Am. J. Epidemiol., 174, 403-411. 